\title{
Efecto del consumo de un complemento alimentario en la concentración plasmática de cinc en adultos mayores residentes en Santiago de Chile
}

\author{
Rosa Isela Ramos $\mathrm{H}^{1, a}$, Eduardo Atalah $\mathrm{S}^{2}$, \\ Carmen U rteaga $R^{2, b}$, Rutila Castañeda $L^{3}$, \\ Maribel 0 rozco L4, Laura Avila ${ }^{5, a}$, Homero Martínez ${ }^{6,7}$. \\ Effect of the consumption of a food \\ supplement on plasma zinc \\ concentrations of free-living \\ Chilean elderly adults
}

Background: Zinc intake is well below recommendation among Chilean free living elderly adults of low socioeconomic level. Aim: To assess the effect of the consumption of a food supplement on plasma zinc concentrations in elderly adults (EA). Material and Methods: Ambulatory EA $(\geq 70)$ with controlled type 2 diabetes mellitus or high blood pressure, ascribed to public family health centers were studied. They were separated in a control and intervention group, without blinding techniques. The intervention group consumed daily $50 \mathrm{~g}$ of a special nutritional supplement prepared as a soup or porridge, provided by the Government, for 3 months. The control group did not receive the supplement. A good compliance with the supplement was defined as a consumption of 7 portions per week. A fasting venous blood sample was obtained to determine plasma zinc at the beginning and end of the study. Results: Forty three supplemented EA aged 76 \pm 5 years (21 women) and 31 non supplemented EA aged $78 \pm 5$ years (20 women), completed 3 months of follow up. Mean compliance with the supplement was 40.5\% (95\% confidence intervals (CI) 40.3-40.6\%). General characteristics of the study subjects upon recruitment were similar, except for the literacy that was higher in the intervention group. We fitted a multiple linear regression model which explained 39\% of the variance, where the consumption of the nutritional supplement increased the concentration of plasmatic zinc by $4.14 \mu \mathrm{g} / \mathrm{dL}$ (95\% CI 0.25-8.02) ( $\mathrm{p} \varangle$ 0.037), after controlling for sex, age, energy, vitamin E and calcium consumption. Conclusions: The consumption of a food supplement significantly increased plasma zinc concentrations in Chilean elderly adults (Rev Méd Chile 2007; 135: 1015-24).

(Key words: Aged; Dietary supplements; Zinc).

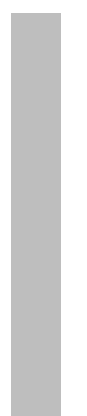

Recibido el 17 de enero, 2006. Aceptado el 6 de marzo, 2007.
Estudio financiado por el Instituto Mexicano del Seguro Social «MSS». Además de
financiamiento obtenido del laboratorio ROCHE en Santiago de Chile, para las determina-
ciones bioquímicas de cinc.
${ }^{1}$ Unidad de Investigación en Epidemiología Nutricional. Instituto Mexicano del Seguro Social.
México, D.F. ${ }^{2}$ Departamento de Nutrición, Facultad de Medicina, Universidad de Chile. Santiago,
Chile. ${ }^{3}$ Unidad de Investigación en Epidemiología Clínica. Instituto Mexicano del Seguro Social.
México, D.F. ${ }^{4}$ Centro Nacional para la Infancia y la Adolescencia. Secretaría de Salud. México
D.F. ${ }^{5}$ Coordinación Clínica de Educación e Investigación en Salud. Instituto Mexicano del Seguro
Social. Cuernavaca, México. ${ }^{6}$ RAND, Santa Mónica, California, EUA. ${ }^{7}$ Subdirección de Investiga-
ción Médica, Hospital Infantil de México «Federico Gómez», Ciudad de México.
aMaestra en Ciencias con mención en Nutrición
bNutricionista

Correspondencia a: Rosa Isela Ramos H. Tabachín \#47, Fraccio-

namiento Brisas de Cuautla, Cuautla, Morelos. CP 62757. Tel./

Fax: Oficina (735) 3016 260. E mail: rosa.ramosh@imss.gob.mx 
L a importancia del cinc para la salud humana se udocumentó por primera vez en 1963. Desde entonces se han conducido múltiples estudios sobre este nutriente, por estar involucrado en funciones bioquímicas importantes y necesarias para mantener la salud a través de todo el ciclo de vida $^{1,2}$. Asimismo, se han identificado factores nutricionales y enfermedades asociadas a la deficiencia de cinc en humanos ${ }^{3}$. Se ha documentado la deficiencia marginal y un estado subóptimo de cinc en diversos grupos poblacionales, tanto en países desarrollados como en desarrollo ${ }^{4}$.

El envejecimiento es un proceso caracterizado por una disminución en el funcionamiento de órganos y sistemas debido a cambios anatómicos y funcionales propios de la edad ${ }^{5}$. Las modificaciones en el aparato digestivo traen como consecuencia una disminución en la capacidad de absorción de micronutrientes ${ }^{6}$. Adicionalmente, se ha documentado que este grupo de edad se encuentra en riesgo para el estado nutricional de cinc, por tener un consumo deficiente de este nutriente $^{5,7,8}$. En América Latina, el porcentaje de población estimada con ingestión inadecuada de cinc fluctúa entre 3,2\% y 55,6\%. En Chile, este porcentaje se ha estimado en $12,5 \%$, considerándose en riesgo bajo de deficiencia de cinc $^{9}$. Estudios realizados en adultos mayores no institucionalizados y de nivel socioeconómico bajo, han mostrado una ingesta de cinc muy por debajo de la recomendación ${ }^{10,11}$.

La deficiencia de cinc es asociada frecuentemente con el envejecimiento y puede generarse debido a una reducción en la ingestión de cinc 0 su biodisponibilidad, problemas psicológicos, actividad física reducida, enfermedades agudas 0 crónicas y consumo de fármacos ${ }^{12}$. La valoración del estado nutricional de cinc sigue siendo un reto, debido a la carencia de indicadores bioquímicos que permitan una rápida y sensible medición de la concentración circulante y de las reservas corporales ${ }^{13}$.

Los programas de apoyo a la alimentación del adulto mayor tienen como objetivo mitigar el riesgo nutricional, buscando mejorar el aporte nutricional a través de acciones que beneficien la salud del adulto mayor ${ }^{14,15}$. El Ministerio de Salud de Chile implementó el Programa de Alimentación Complementaria del Adulto Mayor «ACAM», que provee un complemento alimentario dirigido a población de nivel socioeconómico bajo (Crema Años Dorados). Adicionalmente, incluye actividades preventivas y curativas con el objeto de mantener y mejorar el estado nutricional y la funcionalidad del adulto mayor ${ }^{16}$.

El objetivo de este estudio fue evaluar el efecto del consumo de un complemento alimentario en la concentración plasmática de cinc en adultos mayores beneficiarios de dicho programa.

\section{Material y MÉTodo}

Se llevó a cabo un estudio cuasi-experimental con evaluaciones pre y postintervención, en Centros de Salud Familiar de la ciudad de Santiago de Chile, con duración de tres meses, en la primera mitad de 1999. El tamaño de la muestra estimado fue de 97 sujetos, se perdió $23,7 \%$ de la muestra, quedando conformada la muestra final por 74 adultos mayores. Se calculó el poder estadístico basado en la concentración plasmática de cinc como la principal variable de desenlace, fijando un valor de alfa de 0,05 y $3,0 \mu \mathrm{g} / \mathrm{dL}^{17}$ como diferencia significativa entre grupos para pruebas unilaterales, con un poder estadístico final de $89 \%$.

La población estuvo conformada por adultos mayores de 70 años, ambulatorios, aparentemente sanos o con diabetes mellitus no insulinodependiente o hipertensión arterial controlada, adscritos a cuatro consultorios de atención primaria de Santiago: Santa Julia, Carol Urzúa, Maipú y Ahues. Se excluyeron sujetos con complicaciones como: insuficiencia renal, cáncer, demencia senil o depresión grave.

Mediante un muestreo por conveniencia, se seleccionaron para el Grupo de Intervención (GI) 2 consultorios, entre aquellos inscritos en el programa gubernamental 〈PACAM», que proporciona un complemento alimentario, para ser preparado en forma de sopa o papilla (Crema Años Dorados). El Grupo Control (GC) se reclutó de 2 consultorios que en esa fecha no tuvieron participación en programas de apoyo nutricional o ayuda alimentaria. Para ambos grupos se buscó que en los consultorios estuvieran adscritos adultos mayores con condiciones socioeconómicas similares.

La intervención consistió en el consumo de un complemento alimentario elaborado con harinas de cereales, leguminosas, aceites vegetales y 
verduras deshidratadas, cuya composición nutricional se muestra en el Tabla 1. La indicación fue consumir diariamente una porción de sopa $(50 \mathrm{~g})$, durante 3 meses. Previo al inicio de la intervención, se explicó la forma de preparación, que consistió en agregar $200 \mathrm{~mL}$ de agua caliente hervida. Se consideró $100 \%$ de adherencia cuando el adulto mayor consumió 7 porciones a la semana.

A todos los adultos mayores se les hicieron evaluaciones basales y al final del seguimiento, y se describieron sus características generales, bioquímicas, antropométricas y nutricionales, específicamente la ingestión dietética. Antes de iniciar la suplementación, se tomó una muestra en ayuno de $5 \mathrm{~mL}$ de sangre venosa de los sujetos. Las muestras fueron depositadas inmediatamente en tubos con anticoagulante (heparina sódica libre de cinc, heparina Bestpharma S.A.). Posteriormente se centrifugaron durante 8 min a 3.000 revolucio- nes por minuto (RPM) y finalmente se almacenaron a una temperatura de $-30^{\circ} \mathrm{C}$ hasta el momento de procesarse. Para la determinación del cinc plasmático se utilizó el método de espectrofotometría de absorción atómica ${ }^{18}$, utilizando un espectrómetro Analist 300 (Perkin-Elmer, Norwalk, Ct, USA). Las determinaciones se realizaron en el Laboratorio del Departamento de Nutrición, Facultad de Medicina, Universidad de Chile.

Previo al inicio del estudio, se capacitó y estandanzó a los observadores sobre la técnica y procedimientos de antropometría según lo propuesto por Lohman ${ }^{19}$. El peso y la talla se midieron con una báscula clínica con estadímetro integrado con capacidad de 150 kg, marca Höchstlast. Para medir circunferencia braquial y pliegues cutáneos bicipital (PCB) y tricipital (PCT) se usó una cinta métrica de fibra de vidrio y un calibrador de pliegues cutáneos marca Lange con precisión de $1 \mathrm{~mm}$ y capacidad máxima de $60 \mathrm{~mm}$. La ingestión dietética se obtuvo a través de

Tabla 1. Composición nutricional del complemento alimentario

\begin{tabular}{|lcc|}
\hline Nutrientes & $\begin{array}{c}\text { 1 porción } 50 \mathrm{~g} \\
\text { cantidad }\end{array}$ & \% DDR* \\
\hline Calorías $(\mathrm{kcal})$ & 200 & $\mathrm{NA}^{1}$ \\
Proteínas $(\mathrm{g})$ & 6,5 & $\mathrm{NA}^{1}$ \\
Lípidos $(\mathrm{g})$ & 5,5 & $\mathrm{NA}^{1}$ \\
Hidratos de carbono disponibles $(\mathrm{g})$ & 31,2 & $\mathrm{NA}^{1}$ \\
Fibra dietética total $(\mathrm{g})$ & 3,1 & $\mathrm{NA}^{1}$ \\
Vitamina A $(\mu \mathrm{g} \mathrm{ER})^{2}$ & 120 & 15 \\
Vitamina D $(\mu \mathrm{g})^{3}$ & 0,75 & 15 \\
Vitamina E $(\mathrm{mg}$ ET) & 2 & 20 \\
Vitamina $(\mathrm{mg})$ & 15 & 25 \\
Vitamina $\mathrm{B}_{1}(\mathrm{mg})$ & 0,2 & 12,5 \\
Vitamina $\mathrm{B}_{2}(\mathrm{mg})$ & 0,2 & 12,5 \\
Niacina $(\mathrm{mg} \mathrm{EN})^{5}$ & 2,25 & 12,5 \\
Vitamina $\mathrm{B}_{6}(\mathrm{mg})$ & 0,50 & 25 \\
Folato $(\mu \mathrm{g})$ & 50 & 25 \\
Vitamina $\mathrm{B}_{12}(\mu \mathrm{g})$ & 0,25 & 25 \\
Calcio $(\mathrm{mg})$ & 200 & 25 \\
Fósforo $(\mathrm{mg})$ & 200 & 25 \\
Magnesio $(\mathrm{mg})$ & 75 & 25 \\
Hierro $(\mathrm{mg})$ & 1,4 & 10 \\
Cinc $(\mathrm{mg})$ & 1,5 & 10 \\
\hline
\end{tabular}

*Expresado con relación a la Dosis Diaria Recomendada de 1989.

${ }^{1} \mathrm{NA}=$ No aplica. ${ }^{2} \mu \mathrm{g}$ ER $=$ ug equivalentes de retinol. ${ }^{3} \mu \mathrm{g}=\mathrm{ug}$ de colecalciferol. ${ }^{4} \mathrm{mg}$ ET= mg equivalentes de $\alpha$-tocoferol. ${ }^{5} \mathrm{mg}$ EN $=$ mg equivalentes de niacina. 
una encuesta de recordatorio de 24 horas. Previo a la aplicación de este instrumento, se llevó a cabo una capacitación y estandarización para el uso de medidas caseras, las que se transformaron en gramos y mililitros. La aplicación de la encuesta tuvo una duración promedio de $30 \mathrm{~min}$. El cálculo de calonías y nutrientes se determinó con el programa Nutrition Analysis Software ${ }^{20}$ versión 6,0. Se calculó el cambio $(\Delta)$ para la ingestión de calonías y nutrientes. Inicialmente se llevó a cabo un análisis descriptivo de los datos con exploración de las distribuciones basales. En el caso de datos faltantes, se exploró si esta información estuvo asociada a variables confusoras de los sujetos en estudio. Se identificó un patrón aleatorio de los datos faltantes, sin observarse diferencias estadísticamente significativas entre los grupos.

Para el análisis bivariado, se emplearon los estadísticos de $\mathrm{Chi}^{2}$ para variables categónicas y t de Student para variables cuantitativas. Se estableció la significancia estadística con un valor de $\mathrm{p}<0,05$ para el rechazo de las hipótesis de nulidad. Se ajustó un modelo de regresión lineal múltiple en busca de predictores de la concentración plasmática de cinc al final del estudio, con ajuste de mínimos cuadrados y una inclusión manual escalonada de las variables con base en el criterio de plausibilidad biológica y significancia estadística ( $p \varangle 0,05$ ).

Se evaluó la modificación del efecto a través de la inclusión de variables de interacción. Se presenta el modelo con la mayor capacidad de predicción y se refieren las interpretaciones en unidades de cambio de cinc plasmático $(\mu \mathrm{g} / \mathrm{dL})$. Para el análisis estadístico de la información se utilizó el software STATA/SE ${ }^{21}$ versión 8,0 para Windows. Este estudio fue sometido a la aprobación del Comité de Ética de la Facultad de Medicina de la Universidad de Chile. Se solicitó el consentimiento de todos los adultos mayores, y en caso de aceptación, se pidió que firmaran una hoja de consentimiento informado. Se les dio la certeza que en la presentación de resultados no se permitirá la identificación de ningún sujeto participante y que el uso de los datos sería únicamente con fines de investigación.

\section{Resultados}

Durante el seguimiento hubo una pérdida de 23 adultos mayores, 8 en el grupo de intervención
(GI) y 15 en el grupo control (GC), por inasistencia a su control de salud ( $n=18)$, domicilios falsos $(n=3)$ y hospitalización por fractura de cadera posterior a una caída ( $\mathrm{n}=2)$. De esta manera, se incluyeron en el análisis 74 adultos mayores, 43 en el GI y 31 en el GC. El porcentaje de sujetos con adherencia al consumo del complemento durante el tiempo de intervención fue de 40,5\% (IC 95\% 40,3-40,6\%). Sólo hubo malestares digestivos asociados al consumo del complemento en 2 adultos mayores, y consistieron en meteorismo, flatulencia y diarrea, sin llegar a ser severo en ninguno de los casos.

En la Tabla 2 se presentan las características generales de los participantes en el estudio, por grupo de intervención. Al comparar el alfabetismo entre los grupos se encontró una mayor proporción de analfabetas en el GC (35,5\% vs 13,9\%) ( $p=0,030)$; exceptuando esta condición, las demás variables fueron similares $(p>0,05)$ en ambos grupos. Aunque las diferencias no fueron estadísticamente significativas, llama la atención en el GI, 13,8\% ( $\mathrm{n}=4)$ presentaba DM2, en comparación con 45,5\% ( $\mathrm{n}=10)$ del GC; $62,1 \%$ (n =18) del GI tenía hipertensión arterial, en contraste con $22,7 \%(\mathrm{n}=5)$ en el GC; $7,2 \%(\mathrm{n}=5)$ del GI presentaba DM2 combinada con hipertensión arterial, en comparación con 18,2\% (n $=4)$ en el GC; y 6,9\% (n =2) del GI presentó enfermedades cardiovasculares diferentes a hipertensión arterial, en comparación con 13,6\% ( $\mathrm{n}=3$ ) del GC (resultados no mostrados).

Los indicadores antropométricos en los sujetos de estudio se muestran en la Tabla 3. En la etapa basal, el índice de masa corporal (IMC) fue significativamente mayor en el GC vs GI $\left(29,1 \mathrm{~kg} / \mathrm{m}^{2}\right.$ vs 27,0 $\mathrm{kg} / \mathrm{m}^{2}$, respectivamente; $\mathrm{p}=0,03$ ); una condición similar se observó para el pliegue cutáneo tricipital (PCT) (GI 13,7 mm vs GC 16,5 mm, p=0,03). Al finalizar el estudio, ambos grupos mostraron incremento en este parámetro, algo mayor en el GI, aunque ninguna de las diferencias (basal vs final 0 final entre grupos) fue estadísticamente significativa.

Se analizó la ingestión de nutrientes en la etapa basal y en la final, así como la magnitud del cambio. En la etapa basal, el consumo de calorías fue de $1.298,0$ en el GI y 1.122,0 en el GC ( $p<0,05)$. La magnitud del cambio al final de la intervención fue de $252,0 \pm 517,0$ y $266,0 \pm 357,0 \mathrm{kcal} /$ día, respectivamente, y no mostró diferencias estadísticamente significativas. Al final del estudio, se observó un 
Tabla 2. Características de los adultos mayores por grupo de intervención

\begin{tabular}{|c|c|c|c|c|c|}
\hline \multirow[t]{2}{*}{ Variables } & \multicolumn{2}{|c|}{ GI $n=43$} & \multicolumn{2}{|c|}{$\mathrm{GC} n=31$} & \multirow[t]{2}{*}{ Valor $\mathrm{p}$} \\
\hline & $\mathrm{n}$ & $\%$ & $\mathrm{n}$ & $\%$ & \\
\hline Edad (años) $(\overline{\mathrm{x}} \pm \mathrm{DE})$ & \multicolumn{2}{|c|}{$75,6 \pm 4,5$} & \multicolumn{2}{|c|}{$77,5 \pm 5,3$} & $0,107^{1}$ \\
\hline \multicolumn{6}{|l|}{ Sexo } \\
\hline Masculino & 22 & 51,2 & 11 & 35,4 & \\
\hline Femenino & 21 & 48,8 & 20 & 64,6 & $0,179^{2}$ \\
\hline \multicolumn{6}{|l|}{ Ocupación } \\
\hline Jubilado & 39 & 90,7 & 28 & 90,3 & \\
\hline Inactivo & 4 & 9,3 & 3 & 9,7 & $0,957^{2}$ \\
\hline \multicolumn{6}{|l|}{ Alfabetismo } \\
\hline Sabe leer y escribir & 37 & 86,1 & 20 & 64,5 & \\
\hline Analfabeta & 6 & 13,9 & 11 & 35,5 & $0,030^{2}$ \\
\hline \multicolumn{6}{|l|}{ Antecedentes de morbilidad } \\
\hline Enfermedades asociadas ${ }^{3}$ & 29 & 67,4 & 22 & 70,9 & \\
\hline Aparentemente sanos & 14 & 32,6 & 9 & 29,1 & $0,746^{2}$ \\
\hline \multicolumn{6}{|l|}{ Apetito } \\
\hline Sin cambio & 32 & 74,4 & 25 & 80,7 & \\
\hline Disminuido & 11 & 25,6 & 6 & 19,3 & $0,527^{2}$ \\
\hline \multicolumn{6}{|c|}{ Uso de más de 3 medicamentos 4} \\
\hline Sí & 7 & 16,3 & 5 & 16,1 & \\
\hline No & 36 & 83,7 & 26 & 83,9 & $0,986^{2}$ \\
\hline \multicolumn{6}{|c|}{ Uso de suplementos multivitamínicos } \\
\hline Sí & 7 & 16,3 & 9 & 29,0 & \\
\hline No & 36 & 83,7 & 22 & 71,0 & $0,191^{2}$ \\
\hline
\end{tabular}

${ }^{1}$ Prueba de $t$ de student GI vs GC basal y final. ${ }^{2}$ Prueba de $\mathrm{Chi}^{2} .{ }^{3}$ Diabetes no insulino dependiente e hipertensión. ${ }^{4}$ De acuerdo a MNA ${ }^{35}$.

incremento en la ingesta de vitamina $\mathrm{B}_{6}$ sólo para el GI ( $\mathrm{p}<0,05)$. En la etapa basal la ingestión de hierro fue significativamente mayor en GI (GI 8,5 $\pm 2,9 \mathrm{mg}$ vs GC $7,2 \pm 2,3 \mathrm{mg}$ ), pero el cambio mostrado en la determinación final no mostró diferencia estadísticamente significativa entre grupos. Al inicio del estudio la ingesta de cinc fue menor en el GC, sin tener significancia estadística (Tabla 4).

En la Tabla 5 se muestra la concentración de cinc plasmático basal y al final de la intervención. En la etapa basal se observó una concentración de cinc plasmático un poco más elevada en el GC comparado con el GI; al final de la intervención la concentración de cinc se incrementó en el GI y disminuyó en el GC. Sin embargo, al analizar estos cambios, al interior y entre los grupos, no hubo diferencias estadísticamente significativas.

Al aplicar el modelo multivariado para explicar el incremento en la concentración de cinc plasmáti- co, se evaluó la modificación del efecto a través de la inclusión de variables de interacción, y ninguna mostró un valor estadísticamente significativo. Dada la evidencia de que el contenido de calcio en la dieta puede afectar la absorción de cinc, por la propensión a formar complejos quelados, y que durante la depleción de cinc hay una mala absorción de vitamina $\mathrm{E}$, estas dos variables se incluyeron intencionalmente en el modelo multivariado. Asimismo, el modelo se ajustó por la concentración plasmática de cinc con que iniciaron los sujetos la intervención, sexo, edad y consumo de calorías. Al evaluar la bondad del ajuste hubo una varianza constante de los errores, el valor de la prueba de heterocedasticidad mostró una $\mathrm{Chi}^{2}=0,27$, con un valor de $\mathrm{p}=0,605$ y la capacidad de explicación del modelo fue de $38,50 \%$. Aplicando este modelo, encontramos que el efecto del consumo del complemento alimentario fue la elevación de 4,14 $\mu \mathrm{g} /$ 
Tabla 3. Indicadores antropométricos de los adultos mayores por grupo de intervención

\begin{tabular}{|c|c|c|c|}
\hline Variables & $\begin{array}{c}\text { GI } n=43 \\
\bar{x} \pm \mathrm{DE}\end{array}$ & $\begin{array}{c}\mathrm{GC} n=31 \\
\overline{\mathrm{x}} \pm \mathrm{DE}\end{array}$ & Valor $\mathrm{p}$ \\
\hline \multicolumn{4}{|l|}{ Peso $(\mathrm{kg})$} \\
\hline Basal & $67,2 \pm 7,2^{\mathrm{a}}$ & $70,6 \pm 14,6^{b}$ & $0,252^{1}$ \\
\hline Final & $68,0 \pm 7,0^{\mathrm{a}}$ & $71,1 \pm 14,4^{\mathrm{b}}$ & $0,698^{1}$ \\
\hline Talla (m) & $1,5 \pm 0,1$ & $1,5 \pm 0,1$ & $0,149^{1}$ \\
\hline \multicolumn{4}{|l|}{$\operatorname{IMC}\left(\mathrm{kg} / \mathrm{m}^{2}\right)$} \\
\hline Basal & $27,0 \pm 3,6^{c}$ & $29,1 \pm 4,6^{\mathrm{d}}$ & 0,0371 \\
\hline Final & $27,8 \pm 3,3^{c}$ & $29,3 \pm 4,6^{\mathrm{d}}$ & $0,698^{1}$ \\
\hline Estado nutricional (IMC) ${ }^{2}$ & $\mathrm{n} \quad \%$ & & \\
\hline Enflaquecido $<23,0$ & 9,3 & 3,2 & \\
\hline Normal 23,0-27,9 & 2148,8 & $13 \quad 41,9$ & \\
\hline Sobrepeso $28,0-32,9$ & 34,9 & 1135,5 & $0,360^{3}$ \\
\hline Obesidad $\geq 33,0$ & 7,0 & 19,4 & \\
\hline Circunferencia braquial $(\mathrm{cm})$ & $29,5 \pm 3,0$ & $30,0 \pm 4,0$ & $0,561^{1}$ \\
\hline \multicolumn{4}{|l|}{ Pliegue bicipital (mm) } \\
\hline Basal & $6,2 \pm 3,1 \mathrm{e}$ & $6,6 \pm 2,7^{f}$ & $0,572^{1}$ \\
\hline Final & $7,5 \pm 3,3^{e}$ & $7,7 \pm 2,6^{f}$ & $0,013^{1}$ \\
\hline \multicolumn{4}{|l|}{ Pliegue tricipital (mm) } \\
\hline Basal & $13,7 \pm 5,4^{g}$ & $16,5 \pm 5,4^{h}$ & $0,034^{1}$ \\
\hline Final & $15,4 \pm 6,6 \mathrm{~g}$ & $17,1 \pm 5,8 \mathrm{~h}$ & 0,219 \\
\hline Perímetro muscular braquial (cm) & $25,2 \pm 2,0$ & $24,8 \pm 3,9$ & $0,623^{1}$ \\
\hline Área muscular braquial $\left(\mathrm{mm}^{2}\right)$ & $5.088 \pm 808,9$ & $5.027 \pm 1.821,5$ & $0,862^{1}$ \\
\hline Área grasa braquial $\left(\mathrm{mm}^{2}\right)$ & $1.916,8 \pm 878,9$ & $2.275,4 \pm 819,5$ & $0,076^{1}$ \\
\hline
\end{tabular}

${ }^{1}$ Prueba de $\mathrm{t}$ de Student. ${ }^{2}$ Clasificación del Estado Nutricio según IMC propuesto por el Ministerio de Salud en Chile. ${ }^{3}$ Prueba de $\mathrm{Chi}^{2}$.

aPrueba de $t$ de Student GI basal vs final $p=0,613$. brueba de $t$ de Student GC basal vs final $p=0,876$. CPrueba de $t$ de Student GI basal vs final $p=0,716$. dPrueba de $t$ de Student GC basal vs final $p=0,834$. ePrueba de $t$ de Student GI basal vs final $p=0,063$. Prueba de $t$ de Student GC basal vs final $p=0,104$. gPrueba de $t$ de Student GI basal vs final $p=0,202$. hPrueba de $t$ de Student GC basal vs final $p=0,173$.

Tabla 4. Ingestión de nutrientes en la etapa basal y magnitud del cambio por grupo de intervención

\begin{tabular}{|lcccc|}
\hline \multirow{2}{*}{ Nutrientes } & \multicolumn{2}{c}{ GI $\mathrm{n}=43$} & \multicolumn{2}{c|}{$\mathrm{GC} \mathrm{n}=31$} \\
& Basal & $\Delta$ & Basal & $\Delta$ \\
& $\overline{\mathrm{x}} \pm \mathrm{DE}$ & $\overline{\mathrm{x}} \pm \mathrm{DE}$ & $\overline{\mathrm{x}} \pm \mathrm{DE}$ & $\overline{\mathrm{x}} \pm \mathrm{DE}$ \\
\hline Calorías (kcal) & $1.298 \pm 40^{1}$ & $252 \pm 517$ & $1.122 \pm 330^{1}$ & $266 \pm 357$ \\
Proteínas (g) & $49,4 \pm 16,6$ & $8,8 \pm 25,9$ & $44,7 \pm 19,4$ & $11,8 \pm 21,9$ \\
Hidratos de carbono (g) & $189,2 \pm 60,0$ & $35,2 \pm 69,2$ & $165,2 \pm 42,3$ & $43,3 \pm 53,6$ \\
Lípidos totales (\%) & $38,3 \pm 19,9$ & $9,3 \pm 29,1$ & $32,1 \pm 13,1$ & $4,0 \pm 17,3$ \\
Fibra dietética (g) & $13,6 \pm 7,3$ & $4,6 \pm 8,5$ & $12,0 \pm 6,8$ & $5,5 \pm 8,3$ \\
Vitamina B B $_{6}$ (mg) & $1,0 \pm 0,5$ & $1,5 \pm 1,6^{2}$ & $0,9 \pm 0,5$ & $0,1 \pm 0,5^{2}$ \\
Folato ( $\mu$ g)/d) & $170,3 \pm 108,2$ & $88,9 \pm 190,1$ & $169,9 \pm 138,2$ & $68,8 \pm 139,4$ \\
Vitamina E (mg ET) & $8,0 \pm 4,9$ & $5,2 \pm 6,75$ & $6,8 \pm 3,4$ & $4,9 \pm 4,7$ \\
Calcio (mg) & $492,9 \pm 308,5$ & $167,8 \pm 372,1$ & $460,9 \pm 307,4$ & $142,5 \pm 468,3$ \\
Hierro (mg) & $8,5 \pm 2,91$ & $1,4 \pm 4,6$ & $7,2 \pm 2,3^{1}$ & $2,1 \pm 3,2$ \\
Cinc (mg) & $5,2 \pm 1,7$ & $1,7 \pm 2,8$ & $4,8 \pm 2,8$ & $1,0 \pm 2,6$ \\
\hline
\end{tabular}

${ }^{1}$ Diferencia significativa entre GI vs GC, en la etapa basal ( $\left.p<0,05\right) .{ }^{2} t$ de Student para diferencias en el cambio entre GI vs GC ( $p<0,05)$. ${ }^{\Delta}$ Magnitud del cambio. 
Tabla 5. C aracterísticas del cinc plasmático de los adultos mayores por grupo de intervención

\begin{tabular}{|lccc|}
\hline Variables & GI $\mathrm{n}=43$ & GC $\mathrm{n}=31$ & Valor $\mathrm{p}$ \\
\hline Cinc $\mu \mathrm{g} / \mathrm{dL}(\overline{\mathrm{x}} \pm \mathrm{DE})$ & & & \\
Concentración basal & $82,6 \pm 10,3^{\mathrm{a}}$ & $86,0 \pm 11,6^{\mathrm{b}}$ & $0,206^{1}$ \\
Concentración final & $84,9 \pm 9,1^{\mathrm{a}}$ & $82,1 \pm 10,6^{\mathrm{b}}$ & $0,115^{1}$ \\
\hline
\end{tabular}

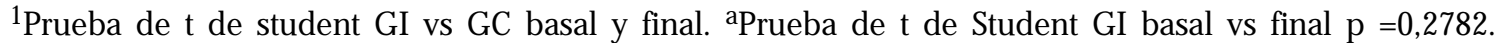
bPrueba de $t$ de Student GC basal vs final $p=0,1806$.

dL en la concentración de cinc plasmático, con un intervalo de confianza al 95\% de 0,25 a 8,02 $\mu \mathrm{g} / \mathrm{dL}$ ( $p=0,037$ ), comparado con el GC y ajustado por sexo, edad, consumo de calorías, vitamina E y calcio (Tabla 6).

\section{DISCUSIÓN}

En los adultos mayores es difícil lograr que la alimentación provea suficientes nutrientes debido a cambios psicológicos, sociales, económicos y de salud. Ante esta problemática, el Ministerio de Salud, preocupado por los adultos mayores, se ha propuesto brindarles atención nutricia a través de la entrega de un complemento alimentario, dado que los alimentos son, de manera incontrovertible, el mejor vehículo para el consumo de nutrientes ${ }^{22}$.
El consumo del complemento alimentario preparado en forma de sopa que fue bien aceptado, con base en el nivel de adherencia $(\approx 40,5 \%)$, proporción similar a la reportada por Bunout ${ }^{23}$.

En relación al incremento en la concentración de cinc plasmático, en la interpretación de estos resultados hay que tomar en cuenta que los adultos mayores son susceptibles a modificaciones en el estado nutricio de cinc, principalmente por cuadros de deficiencia marginal, a pesar de que la deficiencia de cinc plasmático $(<70 \mu \mathrm{g} / \mathrm{dL})$ en la muestra estudiada fue de $8 \%$ (datos no mostrados). Nuestros resultados coinciden con la consideración de que la población chilena está en riesgo bajo para deficiencia de cinc ${ }^{9}$.

Aunque son muchos los factores que intervienen en el estado nutricio de cinc, no podemos soslayar el hecho de que el consumo del complemento alimentario, durante tres meses de inter-

Tabla 6. Resultados de un modelo de regresión lineal múltiple para explicar el papel de las variables de estudio sobre la concentración de cinc plasmático en adultos mayores después de la intervención

\begin{tabular}{|c|c|c|c|}
\hline Variables & Coeficiente $ß$ & IC $95 \% 1$ & Valor $\mathrm{p}$ \\
\hline Complemento alimentario (1 porción $50 \mathrm{~g}$ ) & 4,14 & 0,25 a 8,02 & 0,037 \\
\hline Concentración plasmática inicial de $\mathrm{Zn}^{2}(\mu \mathrm{g} / \mathrm{dL})$ & 0,47 & 0,30 a 0,64 & 0,000 \\
\hline $\operatorname{Sexo}^{3}$ & 1,47 & $-2,66$ a 5,61 & 0,478 \\
\hline Segundo cuartil de edad (75,5 a 79,9 años) & $-7,01$ & $-11,65 a-2,38$ & 0,004 \\
\hline Tercer cuartil de edad ( 80 a 89,9 años) & $-1,90$ & $-6,63$ a 2,83 & 0,425 \\
\hline Cuarto cuartil de edad ( $\geq 90$ años) & $-1,46$ & $-6,09$ a 3,16 & 0,530 \\
\hline$\Delta$ Calorías (kcal) & 0,01 & 0,00 a 0,01 & 0,012 \\
\hline$\Delta$ Vitamina $\mathrm{E}(\mathrm{mg} \mathrm{ET})$ & $-0,44$ & $-0,78$ a $-0,08$ & 0,016 \\
\hline$\Delta$ Calcio $(\mathrm{mg})$ & $-0,01$ & $-0,01$ a 0,00 & 0,053 \\
\hline Constante & 43,45 & & 0,000 \\
\hline $\mathrm{R}^{2}$ & 0,385 & & \\
\hline
\end{tabular}

${ }^{1}$ IC 95\% - Intervalo de Confianza a 95\%. ${ }^{2}$ Zn (Cinc plasmático). ${ }^{3}$ Mujer como categoría de referencia. ${ }^{\Delta}$ Magnitud del cambio 
vención, esté asociado a un incremento de 4,1 $\mu \mathrm{g} / \mathrm{dL}$ en la concentración de cinc plasmático. Se ha documentado que, ante deficiencias marginales de cinc, las concentraciones plasmáticas frecuentemente son normales, por lo que el incremento sérico o plasmático ante una suplementación es la que hace el diagnóstico final del estado nutricio del cinc ${ }^{24-26}$. Como ha sido ya señalado en la literatura, los métodos ultrasensibles basados en la espectrofotometría son más sensibles que los métodos químicos previos, en consecuencia permiten identificar diferencias menores ${ }^{27}$. Esta mayor precisión en la medición del incremento en la concentración cinc, sugiere que el incremento observado en la población estudiada es real, y no se debe al método utilizado.

Por otro lado, llama la atención encontrar una baja ingesta de cinc y niveles plasmáticos en límites relativamente normales. Esta discordancia se podría explicar porque la homeostasis del cinc es mantenida por ajustes en la fracción de absorción del cinc y la excreción intestinal endógena, de manera que las ingestiones de cinc que son sustancialmente menores que las recomendadas pueden ser adecuadas para mantener el contenido de cinc corporal ${ }^{8}$. Adicionalmente, el cinc plasmático, como indicador único, no evalúa en forma precisa el estado nutricio de este oligoelemento, ya que sólo representa $0,1 \%$ del cinc corporal total y se ve alterado solamente cuando los depósitos están considerablemente disminuidos ${ }^{13}$.

El complemento alimentario no indujo cambios significativos en los indicadores antropométricos; resultados similares a los observados en otros estudios donde la intervención tuvo una mayor duración ${ }^{23,28}$. En nuestro estudio, sólo se observó un incremento significativo en el pliegue cutáneo bicipital en el grupo intervenido. El significado biológico de este incremento, podnía estar comprometido por los cambios en los tejidos corporales de los adultos mayores ${ }^{19}$. Por otro lado, varios estudios han sustentado que los mayores cambios en los indicadores antropométricos se presentan en adultos mayores suplementados que de manera inicial tenían bajo peso ${ }^{29,30}$, lo que comesponde al grupo estudiado.

Por los datos obtenidos a través del modelo multivariado, se puede pensar en la formación intestinal de complejos quelados entre el cinc y el calcio, lo que encuentra apoyo en la literatura sobre el potencial efecto inhibidor del calcio sobre la absorción del cinc ${ }^{4}$. Se ha documentado que ante una deficiencia de cinc existe mala absorción de la vitamina E. Es probable que los niveles disminuidos de cinc representen un antioxidante secundario, limitando el daño oxidativo por otras vías. A través de una redistribución del cinc circulante en los tejidos y las células, para la estabilización de membranas o síntesis de prostaglandinas 31,32 .

Aun cuando sería deseable evaluar el efecto del complemento con indicadores de funcionalidad (neurológicos, cognitivos e inmunológicos), la ausencia de estas determinaciones es una limitante en nuestro estudio. En Alemania, un estudio que buscó la correlación entre el estado de cinc y la función inmune en el adulto mayor, documentó que la suplementación oral con cinc mejoró la respuesta inmune en adultos mayores ${ }^{33}$. Asimismo, hubiera sido deseable evaluar la interacción del cinc plasmático con el hierro y el cobre, ya que las dosis altas de cinc (40-50 mg/día) disminuyen la absonción de cobre y predisponen a una deficiencia de este mineral ${ }^{26}$. Sin embargo, en nuestro estudio, el aporte de cinc contenido en el complemento fue de $1,5 \mathrm{mg}$ por porción de $50 \mathrm{~g}$ de alimento. La interacción entre el cinc y el hierro es explicada por la competencia de estos minerales por los transportadores localizados en la cara apical del epitelio del intestino delgado ${ }^{34}$. A causa de que las dosis de hierro y cinc del complemento están por debajo del requerimiento para el adulto mayor, esto no representa un efecto negativo.

En resumen, este estudio llevado a cabo en adultos mayores residentes en Santiago de Chile, fue capaz de evaluar el efecto del consumo de un complemento alimentario sobre la concentración plasmática de cinc, documentando un incremento significativo, que evidencia deficiencia marginal de este nutriente. Esta evaluación es importante, ya que los resultados podrían contribuir a mejorar el programa de suplementación. A pesar de que el consumo del complemento alimentario no tuvo como objetivo el cubrir las recomendaciones diarias de ingestión de cinc y otros nutrientes propuestas para este grupo etáreo, sí mejoró la ingestión de nutrientes, cumpliendo así con su papel de complementar la alimentación. Intervenciones como éstas son necesarias para mejorar el estado nutricio de los adultos mayores, así como su calidad de vida. 
Agradecimientos

Los autores desean externar su agradecimiento a los adultos mayores que participaron, al personal de enfermería y a los directores de

\section{REFERENCIAS}

1. Ma J, Betts N. Zinc and copper intakes and their major food sources for older adults in the 1994-96 continuing survey of food intakes by individuals (CSFII). J Nutr 2000; 130: 2838-43.

2. Wood R. Assessment of Marginal Zinc Status in Humans. J Nutr 2000; 130: 1350S-1354S.

3. Prasad A, Fitzgerald J, Hess J, Kaplan J, Pelen F, DARDENNE M. Zinc deficiency in elderly patients. Nutrition 1993; 9: 218-24.

4. LÖNNERDAL B. Dietary factors influencing zinc absorption. J Nutr 2000; 130: 1378S-1383S.

5. Meertens L, Solano L, PeÑa E. Evaluación del estado de zinc en adultos mayores institucionalizados. Archivos Latinoamericanos de Nutrición 1997; 1997: 311-4.

6. Couzy F, Kastenmayer P, Mansourian R, Guinchard S, Munoz-Box R, DirRen H. Zinc absorption in healthy elderly humans and the effect of diet. Am J Clin Nutr 1993; 58: 690-4.

7. Bales CW, Steinman LC, Freeland-Graves JH, Stone JM, Young RK. The effect of age on plasma zinc uptake and taste acuity. Am J Clin Nutr 1986; 44: 664-9.

8. Pinna K, Woodhouse LR, Sutherland B, Shames DM, King JC. Exchangeable Zinc Pool Masses and Turnover Are Maintained in Healthy Men with Low Zinc Intakes. J Nutr 2001; 131: 2288-94.

9. Нотz C, Brown K. International Zinc Nutrition Consultative Group (IZiNCG) Technical Document \#1. Assessment of the risk of zinc deficiency in populations and options for its control. Toronto, Canadá; 2004.

10. Атаlah E, Benavides X, Avila L, Barahona S, CÁRDENAS A. Características alimentarias de adultos mayores de comunas pobres de la Región Metropolitana. Rev Méd Chile 1998; 126: 489-96.

11. Olivares M, Pizarro F, de Pablo S, araya M, Uauy R. Iron, zinc, and copper: contents in common Chilean foods and daily intakes in Santiago, Chile. Nutrition 2004; 20: 205-12.

12. Finamore A, Devirgilis $C$, Panno D, D'Aquino $M$, Polito A, Venneria E et al. Immune response in cada uno de los consultorios de atención primaria. Así como al Departamento de Posgrado y Postítulo de la Universidad de Chile y al laboratorio ROCHE.

relation to zinc status, sex and antioxidant defence in Italian elderly population: the ZENITH study. Eur J Clin Nutr 2005; 59 (Suppl 2): S68-72.

13. Rosado J. Zinc. In: Bourges H, Casanueva E, Rosado J, editors. Recomendaciones de Ingestión de Nutrimentos para la Población Mexicana. Bases Fisiológicas. México, DF: Editorial Médica Panamericana. Instituto Danone México, DF; 2005. p. 267-272.

14. Consejo Nacional de Política Económica Social, Departamento Nacional de Planeación. Modificaciones a los lineamientos para la operación del programa nacional de Alimentación para el Adulto Mayor Juan Luis Londoño De La Cuesta y la selección y priorización de los beneficiarios. Bogotá, D.C.; 2005.

15. Rivera J, MANDUJANo E. Inseguridad alimentaria y estrategias nutricionales en personas de edad avanzada: experiencias latinoamericanas recientes. Nutrición Clínica 2003; 6: 89-92.

16. Crovetto M, Marchesich R. Perfil Nutricional de Chile. Santiago de Chile; 2001.

17. Zambrano N, Quintero J, Faloue L, Souki A, Arias N, PIÑERO M. Consumo de alimentos y variables bioquímicas: reflejo del estado metabólico y nutricional en un grupo de adultos mayores de vida libre en Venezuela. Archivos Latinoamericanos de Nutrición 1996; 46: 196-202.

18. Smith JJ, Butrimovitz G, Purdy W. Direct measurement of zinc in plasma by atomic absorption spectroscopy. Clin Chem 1979; 25: 1487-91.

19. Lohman T, Roche A, Martorell R. Measurement descriptions and techniques: circumferences. En: Anthropometric standardization reference manual. Illinois: Human Kinetics Publishers; 1988.

20. ESHA R. Nutrition Analysis Software. The Food Processor for Windows. In. 6 ed. Salem, Oregon; 1995.

21. Stata C. STATA/SE version 8.0 for Windows. In. 8.0 for Windows ed. Tx, USA: Stata Corp; 2003.

22. TRIPP F. The use of dietary supplements in the elderly: current issues and recommendations. J Am Diet Assoc 1997; 97: 181S-183S.

23. Bunout D, Barrera G, De ia Maza P, Avendaño M, Gattas V, Petermann M et al. The impact of 
nutritional supplementation and resistance training on the health functioning of free-living Chilean elders: results of 18 months de follow-up. J Nutr 2001; 131: 2441S-2446S.

24. Rosado J. Deficiencia de zinc y sus implicaciones funcionales. Salud Pública de México 1998; 40: 181-8.

25. Brown K, Peerson J, Rivera J, Aluen L. Effect of supplemental zinc on the growth and serum zinc concentrations of prepubertal children: a metaanalysis of randomized controlled trials. Am J Clin Nutr 2002; 75: 1062-71.

26. Olivares M, Castilo Durán C, Arredondo M, Uauy R. Cobre y zinc. In: Gil A, Sánchez de Medina F, Ruiz M, Camarero E, Culebras J, González J et al, editors. Tratado de Nutrición. Madrid: Soc. Española de Nutrición Enteral y Parenteral, Acción Médica SA; 2005. p. 973-996.

27. VaueE B, FalchuK K. The biochemical basis of zinc physiology. Physiol Rev 1993; 73: 79-119.

28. Boukaiba N, Fiament C, Acher S, Chappuis P, Piau A, Fusselier M ET AL. A physiological amount of zinc supplementation: effects on nutritional, lipid, and thymic status in an elderly population. Am J Clin Nutr 1993; 57: 566-72.

29. Lauque S, Arnaud-Battandier F, Mansourian R, Guigoz Y, Paintin M, Nourhashemi et al. Protein- energy oral supplementation in malnourished nursing-home residents. A controlled trial. Age and Ageing 2000; 29: 51-6.

30. Fiatarone Singh M, Bernstein M, Ryan A, O’Neill E, CiEMENTS K, Evans W. The effect of oral nutritional supplements on habitual dietary quality and quantity in frail elders. J Nutr Health Aging 2000; 4: 5-12.

31. Goode H, Purkins L, Heatley R, Kelueher J. The effect of dietary vitamin E deficiency on plasma zinc and copper concentrations. Clin Nutr 1991; 10: 233-5.

32. DiSilvestro RA. Zinc in Relation to Diabetes and Oxidative Disease. J Nutr 2000; 130: 1509S-11.

33. HaAse H, Mocchegiani E, Rink L. Correlation between zinc status and immune function in the elderly. Biogerontology 2006; 7: 421-8.

34. Troost F, Brummer R, Dainty J, Hoogewerff J, BuL V, SARIS W. Iron supplements inhibit zinc but not copper absorption in vivo in ileostomy subjects. Am J Clin Nutr 2003; 78: 1018-23.

35. Velias B, Guigoz Y, Garky P, Nourhashemi F, Bennahum D, Lauque $S$ et al. The Mini Nutritional Assessment (MNA) and its use in grading the nutritional state of elderly patients. Nutrition 1999; 15: 116-22. 